\title{
Alternate Pathways of Cerebroside Catabolism
}

YUH-NAN LIN and NORMAN S. RADIN, Mental Heal th Research Institute,

University of Michigan, Ann Arbor, Michigan 48104

\begin{abstract}
A search was made for new degradative pathways for glucosyl and galactosyl ceramides in an effort to explain the failure of these lipids to accumulate in the brains of children with Krabbe's or Gaucher's disease. Using various buffers and incubation conditions, we tested brain homogenates from 12 day old rats with the stearate-labeled and galactoselabeled lipids. No evidence for direct deacylation (and formation of psychosines) could be obtained, nor was there any evidence for transacylation of sphingosyl phosphoryl choline or oxidation of the 6 position of the galactose moiety. Two new derivatives of galactosyl ceramide were observed, possibly fatty acid esters of unknown polar compounds. It is tentatively proposed that the etiology of infantile Krabbe's and Gaucher's diseases involves, not an accumulation of galactosyl and glucosyl ceramides, with consequent formation of toxic products, but rather malfunction of some other role of the corresponding glycosidases.
\end{abstract}

\section{INTRODUCTION}

The only known pathway for catabolism of glucosyl and galactosyl ceramides is through two $\beta$-glycosidases $(1,2)$. In the case of the galactosidase, the enzyme appears to be quite specific and can be distinguished from a number of other $\beta$-galactosidases $(1,3,4)$. It does seem to attack galactosyl sphingosine (5-7), a lipid which hypothetically could be derived from galactosyl ceramide via an amidase. Glucocerebrosidase probably is not the only mammalian $\beta$-glucosidase, judging from comparisons between aryl glucoside and ceramide glucoside as substrates $(2,8)$.

In Krabbe's disease (infantile globoid leukodystrophy), the primary genetic lesion appears to be in the cerebroside galactosidase (9). The striking feature of this disease is that there is no accumulation of galactosyl ceramide in brain; but instead there is a marked, fatal lack of brain development. Miyatake and Suzuki (5) have made the suggestion that the galactosyl ceramide is converted to galactosyl sphingosine, which may be highly toxic and thus cause the fatal failure to develop. This alternative cata- bolic product is assumed to be so toxic that detectable concentrations fail to accumulate. The failure of cerebroside to accumulate does not seem to be due to loss of the synthetic enzymes which make the ceramides and galactosyl ceramides (10).

A similar situation could be postulated in the infantile form of Gaucher's disease, which seems to be due to a severe lack of glucosidase (11). The young brain is an active manufacturer of glucosyl ceramide for use in making gangliosides; and there seems to be an active catabolism of gangliosides, so one should expect the brain to accumulate much glucosyl ceramide in infantile Gaucher's disease. Instead there seems to be no accumulation, and the brain simply fails to mature, degenerating to a fatal condition, as in the Krabbe disorder. (Although a few cases of the infantile form of the disease have been found to accumulate some glucocerebroside in the brain, the lipid appears to be in unusual cells, rather than in neurons [12]). Thus one might suggest that glucosyl ceramide could be converted to glucosyl sphingosine, a potentially highly toxic substance.

While our early studies on galactosyl ceramide break down (1) gave negative evidence for fatty acid release (except for a bit that could be attributed to ceramidase action), no such examination appears to have been made in the case of glucosyl ceramide. Consequently we have reopened the question with the aid of cerebrosides labeled in the galactose and fatty acid moieties by incubating them with brain homogenates and examining the radioactive products. No evidence for fatty acid release could be obtained, but some conversion of galactosyl ceramide to two unknown polar substances was observed.

\section{MATERIALS AND METHODS}

\section{Substrates}

The synthesis of the two cerebrosides labeled with $[1-14 \mathrm{C}]$ stearic acid has been described before $(13,14)$. Examination of the galactosyl ceramide by thin layer chromatography (TLC) radioautography showed only one spot, and elution of this spot and other plate regions indicated a radiopurity of over $97 \%$. The glucosyl ceramide showed a slight radioactive streak above and below the cerebroside spot and a minor spot just below the main spot, 
TABLE I

Fatty Acid Fraction Produced from Incubations with Brain Homogenate and Stearate-Labeled Cerebrosides

\begin{tabular}{|c|c|c|c|c|}
\hline \multirow[b]{3}{*}{ Substrate } & \multirow[b]{3}{*}{ Buffer } & \multicolumn{3}{|c|}{$\begin{array}{l}\text { Per cent conversion of } \\
\text { labeled substrate }\end{array}$} \\
\hline & & \multicolumn{2}{|c|}{ Emulsion } & \multirow{2}{*}{$\frac{\text { On Celit }}{(32 \mathrm{mg}}$} \\
\hline & & $(8 \mathrm{mg})$ & $(32 \mathrm{mg})$ & \\
\hline Galactosyl ceramide & Phosphate 7.0 & $0.03^{a}$ & $0.03^{a}$ & $0.04^{a}$ \\
\hline \multirow[t]{9}{*}{$200,000 \mathrm{cpm}$} & Citrate 4.0 & .03 & .04 & .04 \\
\hline & Citrate 5.0 & .03 & .04 & .04 \\
\hline & Citrate 6.2 & .03 & .06 & $\cdots$ \\
\hline & Phosphate 6.0 & .04 & .04 & .05 \\
\hline & Phosphate 7.0 & .04 & .05 & .04 \\
\hline & Phosphate 8.0 & .04 & .05 & --- \\
\hline & Tris 7.2 & .04 & .05 & $\cdots$ \\
\hline & Tris 8.0 & .06 & .06 & .05 \\
\hline & Tris 8.9 & .05 & .04 & .05 \\
\hline Glucosyl ceramide $b$ & Citrate 5.0 & $1.76^{\mathrm{a}}$ & & $0.20^{\mathrm{a}}$ \\
\hline \multirow[t]{6}{*}{$200,000 \mathrm{cpm}$} & Citrate 4.0 & 1.39 & & .28 \\
\hline & Citrate 5.0 & 5.78 & & .28 \\
\hline & Phosphate 6.0 & 4.33 & & .50 \\
\hline & Phosphate 7.0 & 3.79 & & .35 \\
\hline & Tris 8.0 & 1.38 & & .34 \\
\hline & Tris 8.9 & 1.42 & & .34 \\
\hline
\end{tabular}

a Data from boiled enzyme. These numbers were not subtracted from the others.

b The glucocerebroside used to make the emulsion had not been purified recently and yielded a high blank in the fatty acid fraction.

which could be the dihydrosphingosine form. The radiopurity, taking the minor spot to be an impurity, was $96 \%$. Galactosyl ceramide labeled in the 6 position of the sugar with tritium was made by the borohydride method (15). Sphingosyl phosphoryl choline hydrochloride was made from sphingomyelin by hydrolysis in methanol-water- $\mathrm{HCl}(16)$ and purified with a long silica gel column.

The cerebrosides were tested as emulsions in Tween 20, Myrj 59, and Na taurocholate (17); tris oleate was omitted from the emulsion.

\section{Enzyme Test Procedure}

Some incubations were performed with 0.4 $\mathrm{ml}$ cerebroside emulsion ( $0.1 \mathrm{mg}$ cerebroside), 0.1 mmole buffer, 0.2 moles dithiothreitol, and brain homogenate in a total volume of 1 $\mathrm{ml}$. The homogenate was made from brains of 12-day-old Sprague-Dawley rats in 11 volumes cold water. The homogenate was stored in portions in the freezer, and each series was carried out on material which had been thawed only once. The rats were chosen to be at the pre-myelination age, which corresponds more closely to the human disorders and which minimizes dilution of the radioactive substrate by endogenous cerebroside.

Some incubations were carried out in the absence of detergent, and the cerebroside then was coated onto $50 \mathrm{mg}$ Celite Analytical Filter
Aid by evaporation from solution. To this was added 0.1 mmole buffer, $0.2 \mu$ mole dithiothreitol, and brain homogenate, as above.

Where sphingosyl phosphoryl choline was added to the mixture, $0.2 \mathrm{mg}$ lipid was applied to the bottom of the incubation tube by evaporation from solution prior to the other additions. The lipid dissolved readily in each buffer.

Buffers used were Na-citrate, Na-phosphate, and Tris- $\mathrm{HCl}$ at $\mathrm{pH}$ noted in the tables. The tubes were shaken $1 \mathrm{hr}$ at $37 \mathrm{C}$, then $20 \mathrm{ml}$ chloroform-methanol were added. The mixture was acidified with $4 \mathrm{ml} 0.5 \mathrm{M}$ citric acid, and the resultant upper layer was discarded. The lower layer was washed with $10 \mathrm{ml}$ methanolwater $(1: 1)$ and filtered to remove protein. The filtrate was evaporated to dryness under vacuum with the aid of benzene additions, taken up in chloroform, and applied to a silica gel column $(0.4 \times 6 \mathrm{~cm}, 0.32 \mathrm{~g})$. A fatty acid fraction was eluted with $10 \mathrm{ml}$ chloroform, a cerebroside fraction with $10 \mathrm{ml}$ chloroformmethanol (90:10), and a polar lipid fraction with $10 \mathrm{ml}$ chloroform-methanol $(1 ; 2)$. Portions of the first and third fractions were counted and examined by TLC radioautography.

\section{RESULTS}

Examination of the first column fraction 
TABLE II

Fatty Acid Fraction Produced from Incubations with Brain Homogenate and Labeled Glucocerebroside in the Presence of Glucosidase Inhibitors

\begin{tabular}{llc}
\hline Buffer & \multicolumn{1}{c}{ Inhibitor } & $\begin{array}{c}\text { Per cent conversion } \\
\text { of cerebroside }\end{array}$ \\
\hline Citrate $5.0^{\mathrm{a}}$ & None & 5.37 \\
& None (boiled enzyme) & 1.76 \\
& $5 \mu \mathrm{M}$ hexyl glucosyl sphingosine & 2.05 \\
Phosphate $6.0^{\mathrm{b}}$ & $5 \mathrm{mM} \delta$-gluconolactone & 1.94 \\
& None & 0.33 \\
& None (boiled enzyme) & .26 \\
& $20 \mu \mathrm{M}$ hexyl glucosyi sphingosine & .17 \\
\hline
\end{tabular}

${ }^{a}$ These incubations run with cerebroside emulsion and $16 \mathrm{mg}$ brain.

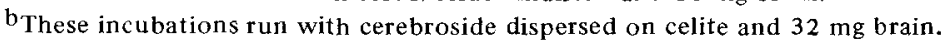

from galactocerebroside incubations showed very slight formation of free fatty acid (Table I). While radioactive ceramide was formed in the incubations containing detergents and taurocholate, there was evidently insufficient ceramide for further conversion at an appreciable speed into free fatty acid. In the case of glucocerebroside, the first column fraction did show an appreciable amount of radioactivity, especially at $\mathrm{pH} 5$ with the emulsified substrate and $\mathrm{pH} 6$ with the nonemulsified substrate. The formation of fatty acid was much less in the tubes lacking taurocholate and detergents, which can be explained by the well-established need for these adjuncts in the normal catabolic pathway (via glucosidase and ceramidase). Evidently glucosidase was sufficiently active in these preparations to raise the ceramide level to the point where ceramidase action could be observed.

Further evidence for this interpretation was obtained by incubating labeled glucocerebroside in the presence of inhibitors of glucosidase (Table II). Both inhibitors used brought down the yield of labeled fatty acid close to the blank level.

Assuming the inhibitors also do not block the hypothetical amidase, we conclude that the radioactivity observed without inhibitors is due to a rapid hydrolysis of glucocerebroside to ceramide, and then to sphingosine and free fatty acid.

The polar lipid fraction (Table III) showed some conversion of galactocerebroside to more polar material, particularly at pH 6 and 8 with emulsions or plain cerebroside. This conversion was greater in tubes containing more homogenate and generally seemed higher in the absence of detergents. Radioautography of a TLC plate (silica gel, chloroform-methanolwater $[117: 45: 8]$ ) showed that this fraction contained a radioactive band at the cerebroside position $\left(R_{f} 0.61\right)$, evidently due to some trailing from the second column eluent, and two bands at $R_{f} 0.52$ and 0.38 . When this examination was carried out with the same column fraction after alkaline methanolysis at room temperature for $1 \mathrm{hr}(18)$, the cerebroside band was found to be unchanged, but the two lower bands were now even lower, with $R_{f} 0.29$ and 0.24 . From this it would appear that the two metabolic products were fatty acid esters of some polar cerebroside derivative. The positions of the methanolyzed compounds did not correspond to any known galactosyl ceramide derivatives, including galactosyl galactosyl ceramide. The lower band corresponded to the faster region of cerebroside sulfate or lecithin.

Some conversion of glucocerebroside to polar material also was observed in the absence of detergent (Table III) but was not characterized further, because we assumed that this was a series of ganglioside intermediates made from endogenous sugar donors. The glucosidase inhibitors had no effect on this conversion.

Incubations also were carried out with stearate-labeled galactosyl ceramide and unlabeled sphingosyl phosphoryl choline (lysosphingomyelin) on the theory that the cerebroside is not degraded by an amidase but by a specific fatty acyl transferase which could form galactosyl sphingosine and sphingomyelin. Here too there was some conversion to polar lipid, appearing in the third column eluent, but this was to the same extent as that found without the lysosphingomyelin. TLC radioautography showed the same labeled bands without any sign of radioactivity in the sphingomyelin.

Another possible catabolic route for galactocerebroside was tested by incubations with stearoyl $\left[6-{ }^{3} \mathrm{H}\right]$ galactosyl sphingosine, dispersed on Celite or emulsified, as described 
TABLE III

Polar Lipid Fraction Produced by Incubations with Brain Homogenate and Stearate-Labeled Cerebrosides

\begin{tabular}{|c|c|c|c|c|}
\hline \multirow[b]{3}{*}{ Substrate } & \multirow[b]{3}{*}{ Buffer } & \multicolumn{3}{|c|}{$\begin{array}{l}\text { Per cent conversion of } \\
\text { labeled substrate }\end{array}$} \\
\hline & & \multicolumn{2}{|c|}{ Emulsion } & \multirow{2}{*}{$\frac{\text { On Celite }}{(32 \mathrm{mg})}$} \\
\hline & & $(8 \mathrm{mg})$ & (32 mg) & \\
\hline \multirow[t]{10}{*}{ Galactosyl ceramide } & Phosphate 7.0 & $0.24^{\mathrm{a}}$ & $0.36^{\mathrm{a}}$ & $0.84^{\mathrm{a}}$ \\
\hline & Citrate 4.0 & .20 & .84 & .65 \\
\hline & Citrate 5.0 & .22 & .58 & .61 \\
\hline & Citrate 6.2 & .17 & 1.10 & -- \\
\hline & Phosphate 6.0 & .32 & 1.28 & 2.15 \\
\hline & Phosphate 7.0 & .32 & .97 & 1.26 \\
\hline & Phosphate 8.0 & .33 & .86 & -- \\
\hline & Tris 7.2 & .31 & .65 & --- \\
\hline & Tris 8.0 & .20 & 1.62 & 1.06 \\
\hline & Tris 8.9 & .24 & .71 & 1.81 \\
\hline \multirow[t]{11}{*}{ Glucosyl ceramide ${ }^{b}$} & Citrate 5.0 & $2.76^{\mathrm{a}}$ & & $1.42^{\mathrm{a}}$ \\
\hline & Citrate 4.0 & 1.89 & & 1.72 \\
\hline & Citrate 5.0 & 1.48 & & 1.71 \\
\hline & Phosphate 6.0 & 1.74 & & 2.64 \\
\hline & Phosphate 7.0 & 2.09 & & 1.94 \\
\hline & Tris 8.0 & 2.12 & & 2.41 \\
\hline & Tris 8.9 & 1.95 & & 1.50 \\
\hline & Phosphate 6.0 & & & $.68^{\mathrm{a}}$ \\
\hline & Phosphate 6.0 & & & 1.12 \\
\hline & Phosphate 6.0 & & & $1.28^{\mathrm{c}}$ \\
\hline & Phosphate 6.0 & & & $1.04 \mathrm{~d}$ \\
\hline
\end{tabular}

\footnotetext{
a Data from boiled enzy me.

${ }^{b}$ The glucocerebroside used to make the emulsion had not been purified recently and yielded a high blank in the polar lipid fraction.

c Incubation included $20 \mu \mathrm{M}$ hexyl glucosyl sphingosine.

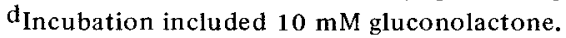

above. After incubating the mixture for $2 \mathrm{hr}$ with brain at $\mathrm{pH} 6$ (phosphate), 7.2 and 8 (Tris), we lyophilized the suspension and measured the radioactivity in the collected water. (In the case of the samples containing Celite, the powder had to be removed before lyophilization to prevent flaking and carryover into the condenser.) In no case was tritium found in the water, indicating a lack of a dehydrogenase that could attack the 6 position of the sugar.

\section{DISCUSSION}

Disregarding the obvious dangers of reporting negative findings and of not trying every possible buffer and incubation medium, we conclude that neither glucosyl nor galactosyl ceramide is metabolized by young brain to form the corresponding hexosyl sphingosine. Our finding of hitherto undetected galactocerebroside derivatives of a polar type raises the possibility that these are toxic in the brain of the Krabbe child, and that their accumulation has not been noticed in previous examinations of the brain lipids. More likely, in our opinion, the seriousness of infantile Krabbe's and Gaucher's diseases is due to malfunction of a thus far unknown role of the cerebroside hydrolases. Preliminary evidence with a glucosidase inhibitor and cultured brain tumor cells suggests a role in membrane transport (19).

The observations that hexosyl sphingosines can be formed enzymatically from the free base and UDP-Hexose $(20,21)$ and that the hexosyl sphingosines can be hydrolyzed to free base and hexose are attributed to nonspecificity on the part of the cerebroside synthetases and hydrolases. Support for this interpretation comes from the fact that the "unnatural" reactions are somewhat slower than the "natural" ones and the fact that the hydrolase activity toward galactosyl sphingosine is severely deficient in Krabbe's disease (5).

\section{ACKNOWLEDGMENTS}

This work was supported by grant NS-03192 from the National Institute of Neurological Diseases and Stroke. D. Greenblatt made the erythro isomer of lysosphingomyelin for this work, and $\mathrm{K}$. Warren made $\mathrm{N}$-(n-hexyl)-O-glucosyl sphingosine.

\section{REFERENCES}

1. Hajra, A.K., D.M. Bowen, Y. Kishi moto, and N.S. 
Radin, J. Lipid Res. 7:379 (1966).

2. Brady, R.O., J. Kanfer, and D. Shapiro, J. Biol. Chem. 240:39 (1965).

3. Bowen, D.M., and N.S. Radin, J. Neurochem. $16: 501$ (1969).

4. Dawson, G., J. Lipid Res. 1 3:207 (1972).

5. Miyatake, T., and K. Suzuki, J. Biol. Chem. 247:5398 (1972).

6. Bowen, D.M., and N.S. Radin, Biochim. Biophys. Acta 152:599 (1968).

7. Brady, R.O., A.E. Gal, J.N. Kanfer, and R.M. Bradley, J. Biol. Chem. 240:3766 (1965).

8. Ho, M.W., J.S. O'Brien, N.S. Radin, and J.S. Erickson, Biochem. J. 131:173 (1973).

9. Suzuki, K., and Y. Suzuki, Proc. Nat. Acad. Sci. 66:302 (1970).

10. Radin, N.S., R.C. Arora, M.D. Ullman, A.L. Brenkert, and J. Austin, Res. Comm. Chem. Pathol. Pharmacol. 3:637 (1972).

11. Brady, R.O., J.N. Kanfer, R.M. Bradley, and D. Shapiro, J. Clin. Invest. 45:1112 (1966).

12. Svennerholm, L., in "Inborn Disorders of Sphingolipid Metabolism," Edited by S.M. Aronson and
B.W. Volk, Pergamon Press, Oxford, England, 1967, p. 173.

13. Erickson, J.S., and N.S. Radin, J. Lipid Res. 14:133 (1973)

14. Kopaczyk, K.C., and N.S. Radin, Ibid. 6:140 (1965).

15. Radin, N.S., in "Methods of Enzymology," Vol. 28, Edited by V. Ginsburg, Academic Press, New York, N.Y., 1972 , p. 300.

16. Gaver, R.C., and C.C. Sweeley, J AOCS 42:294 (1965).

17. Radin, N.S., and R.C. Arora, J. Lipid Res. $12: 256$ (1971).

18. Kishimoto, Y., W.E. Davies, and N.S. Radin, Ibid. 6:525 (1965).

19. Dawson, G., A.C. Stoolmiller, and N.S. Radin, Trans. Amer. Soc. Neurochem. 4:92 (1973).

20. Cleland, W.W., and E.P. Kennedy, J. Biol. Chem. $235: 45$ (1960).

21. Curtino, J.A., and R. Caputto, Lipids 7:525 (1972).

[Received July 9, 1973] 